\title{
Mechanical and tribological characterization of a thermally actuated MEMS cantilever
}

\author{
Marius Pustan · Véronique Rochus • \\ Jean-Claude Golinval
}

Received: 10 March 2011/ Accepted: 28 December 2011

(C) Springer-Verlag 2012

\begin{abstract}
The temperature effect on the mechanical and tribological behaviors of a microelectromechanical systems cantilever is experimentally investigated using an atomic force microscope. A nonlinear variation of the bending stiffness of microcantilevers as a function of temperature is determined. The variation of the adhesion force between the tip of atomic force microscope (AFM) probe $\left(\mathrm{Si}_{3} \mathrm{~N}_{4}\right)$ and the microcantilever fabricated in gold is monitored at different temperatures. Using the lateral mode operation of atomic force microscope, the influence of temperature on friction coefficient between the tip of AFM probe and microcantilever is presented. Finite element analysis is used to estimate the thermal field distribution in microcantilever and the axial expansion.
\end{abstract}

\section{Introduction}

Thermal microelectromechanical systems (MEMS) can be used either as actuators or as sensors. This paper deals with determination of mechanical and tribological characteristics of microcomponents (e.g. MEMS thermal actuators). Many MEMS devices such as thermal actuators, thermal flow sensors, micro-hotplate gas sensors, and tunable

\footnotetext{
M. Pustan ( $\square)$

Technical University of Cluj-Napoca,

Bd. Muncii 103-105, Cluj-Napoca, Romania

e-mail: Marius.Pustan@omt.utcluj.ro

V. Rochus

IMEC, Kapeldreef 75, 3001 Leuven, Belgium

J.-C. Golinval

LTAS-Liège, University of Liège,

Chemin Des Chevreuils 1, 4000 Liege, Belgium
}

optical filters are based on thermo-mechanical coupling (Lobontiu and Garcia 2004). Thermal actuators have several particular applications in inkjet devices, thermal relay and shape memory alloy. Moreover, they are employed in linear and rotary microengines providing large linear motion such that they are integrated with compliant mechanisms to increase their displacement range for different applications (Chu and Gianchandani 2003; Park et al. 2001; Zhu et al. 2006). Thermocouples are used in a wide variety of MEMS sensors, from temperature sensor to thermal flow sensor.

Depending on their actuation principle, MEMS actuators are classified into four main groups: electrostatic, electromagnetic, piezoelectric and thermal (Lobontiu and Garcia 2004). Thermal actuators basically convert thermal energy into mechanical motion. This type of actuation has the advantage of producing relatively large force and displacement compared to electrostatic actuation (Zhu et al. 2006; Geisberger et al. 2003). Moreover thermal actuators are usually simpler, more reliable and easier to fabricate using surface micromachining processes (Paryab et al. 2009). However, these force performances cost a very large input of energy and are performed at very low operating frequency due to the time response to reach thermal equilibrium (Rebeiz 2003; Yang et al. 2008). The heating and cooling times depending on the actuator geometry and materials properties, the power consumption and thermal loss can be reduced by optimizing the structural design of the actuator and by choosing the appropriate material.

Thermal actuators are usually used in transduction applications, which are based on in-plane relative motion. Such motion can be easily performed with microcantilevers. The MEMS cantilevers can operate individuallywith no other accompanying structural component—or can be incorporated into more complex configurations. These 
microcomponents can operate as sensors, actuators or as simple flexible joints in compliant microdevices (Lobontiu and Garcia 2004; Pustan et al. 2007). For these reasons, the structures studied here are microcantilevers.

In order to enhance the design of these devices and to increase their reliability and performance, mechanical and tribological characteristics of sensing/acting microcomponents under the thermal operating conditions must be determined experimentally. In this paper, experimental tests performed using an atomic force microscope (AFM) are presented with the aim of characterizing mechanically and tribologically MEMS thermal components. These actuators are characterized here by their in-plane motion due to thermal expansion and out-of-plane bending. The thermal expansions of microcomponents have an influence on displacement of active elements and on mechanical response of actuators. The principle of axial thermal expansion is described in Sect. 2 of paper, and the relation for bending stiffness is given. Experimental investigations of axial expansions of a microcantilever as a function of temperature are performed using the atomic force microscope AFM scanning mode at the free-end of sample. This work is presented in Sect. 3.1 where different extended positions of the free-end of a gold microcantilever were measured for a temperature range between 20 and $100^{\circ} \mathrm{C}$. After each increase of temperature, the bending stiffness of sample is also determined with the AFM bending mode. The method used to perform these experimental tests is described in Sect. 3.2 as well as the dependence between stiffness and temperature. A nonlinear variation of the bending stiffness of a gold cantilever as a function of temperature is obtained. Section 3.3 presents the tribological analysis of thermal components which implies the estimation of the thermal effects on the adhesion force and atomic friction. The friction between sample and the tip of AFM probe is determined using the AFM lateral force mode. Finite element analysis is used to predict the distribution of the thermal field in sample and the axial expansion of the free-end of microcantilever. The results of finite element analysis are presented in Sect. 4 of paper.

\section{Theoretical formulas for thermal expansion and bending stiffness of a microcantilever}

\subsection{Axial expansion of a thermal actuated microcantilever}

The principle of thermal actuation of a microcantilever is presented in Fig. 1. The beam with an initial length $l_{0}$ is supposed to be deformed in the $x$ direction due to the thermal field. The final length of beam $l$ depends on the temperature $T$ and is calculated as:
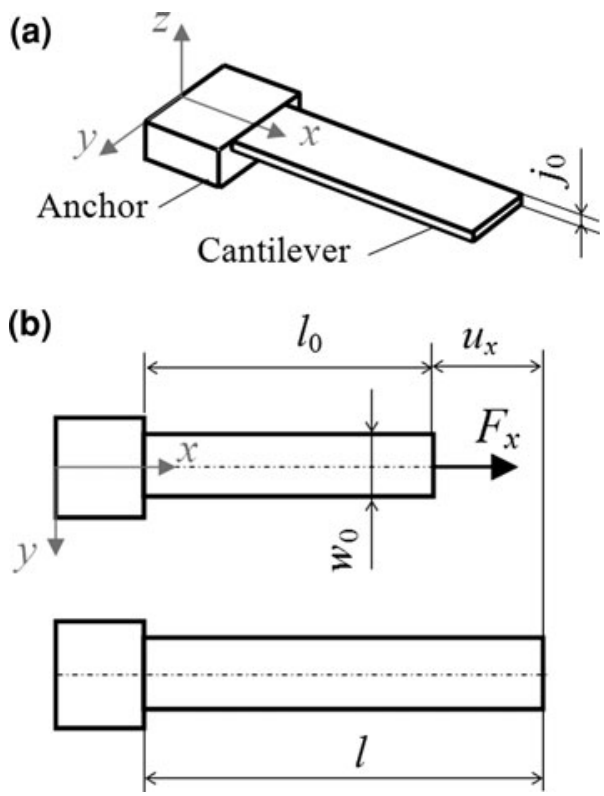

Fig. 1 Fixed-free bar expanding at increase of temperature: a 3D view of cantilever, $\mathbf{b}$ top-view of cantilever at initial and after thermal expansion

$l=l_{0}+u_{x}=l_{0}(1+\alpha \cdot \Delta T)$

where, the material's thermal expansion coefficient in the longitudinal direction, which couples changes of length with changes of temperature is given by

$\alpha=\frac{1}{l_{0}} \cdot \frac{u_{x}}{\Delta T}$

and $u_{x}$ is the axial displacement of the beam and $\Delta T$ is the temperature variation.

This simplest thermal actuator as the free-end of a cantilever can be coupled to a complex microdevice at a part where actuation is needed. The thermal displacement $u_{x}$ (Fig. 1) can also be produced by an equivalent force that acts at the free-end, and which is:

$F_{x}=E \cdot A \cdot \frac{u_{x}}{l_{0}}=E \cdot A \cdot \alpha \cdot \Delta T$

where, $E$ is the material Young's modulus and $A$ is the cross-section area. The output performance in terms of force or pressure of the thermal actuator, such as the simple thermal bar, depends on the load to overcome. If an external load $F_{\text {ext }}$ is applied opposing the thermal expansion, the total displacement of the end of beam is the difference of two opposing deformations, namely:

$u_{x}=\alpha \cdot l_{0} \cdot \Delta T-\frac{F_{e x t} \cdot l_{0}}{E \cdot A}$

relation (4) can be rewritten in the form:

$F_{\text {ext }}=E \cdot A \cdot \alpha \cdot \Delta T-\frac{E \cdot A \cdot u_{x}}{l_{0}}$ 


\subsection{Bending effect of a thermal microcantilever}

The bending stiffness of a cantilever as a function of temperature, when the force is applied at free-end of sample (Fig. 2), can be written as:

$k_{z}=\frac{E w_{0} j_{0}^{3}(1+\alpha \Delta T)}{4 l_{0}^{3}}$

and the force in $z$ direction depending on the bending stiffness of the beam is

$F_{z}=\frac{E w_{0} j_{0}^{3}(1+\alpha \Delta T)}{4 l_{0}^{3}} u_{z}$

where, $u_{z}$ is the bending displacement of the free-end of microcantilever (Fig. 2), $w_{0}$ the initial width and $j_{0}$ is the initial thickness of sample as shown in Fig. 1.

A thermal phenomenon introduces softening due to Young's modulus-temperature relation and a thermal relaxation which affects the rigidity of material (Tadayon et al. 2006), less force is needed to deflect the microcantilever if temperature increases to produce the same displacement as at the initial temperature. In a case of a thermoelastic microcantilever under bending, the relaxation of Young's modulus has to be considered (Lifshitz and Roukes 2000; Lobontiu 2007). Experimentally, the dependence between force $F_{\mathrm{z}}$ and displacement in $z$ direction of a microcantilever (Fig. 2) can be determined and the Young's modulus-temperature dependence can be estimated based on (7) as:

$E=\frac{4 l_{0}^{3}}{w_{0} j_{0}^{3}(1+\alpha \Delta T)} \cdot \frac{F_{z}}{u_{z}}$

\section{Experimental investigations of a thermally actuated microcantilever}

\subsection{Thermal expansion measurement} of a microcantilever using atomic force microscope

This section presents the thermal expansion measurements of a clamped-free microcomponent as a function of temperature. In our experiments, an AFM was used to perform the measurements. Indeed, it can directly display and measure the axial expansion of sample. A mini hotplate,

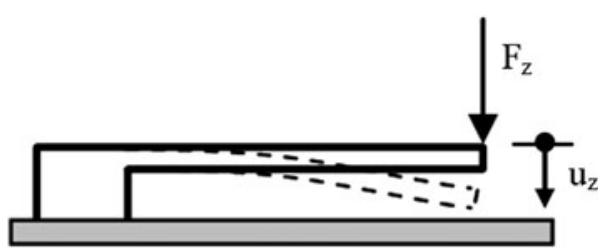

Fig. 2 Bending of a microcantilever beam in $z$ direction with a temperature range from 20 to $100^{\circ} \mathrm{C}$ and a temperature control resolution of $0.1^{\circ} \mathrm{C}$, was placed under the beam anchor to change and control the temperature of microcantilever. The total axial expansion of microcantilever depends on the thermal expansion of the flexible part and on the thermal expansion of the beam anchor. If the microcantilever is used as a thermal actuator, the interest is to evaluate the thermal axial expansion of its free-end (as the sum between the axial expansion of the flexible part and the expansion of the beam anchor) that can be coupled to a complex microdevice at a part where the actuation is needed. Consequently, only the thermal displacements in $x$ direction (Fig. 1) of the beam free-end were AFM monitored. To measure the axial expansion of microcantilever at different temperatures, a scanning zone is selected at its free-end, as presented in Fig. 3.

Thermal displacements of the free-end of microcantilever are determined using the AFM scanning mode. During experimental tests the following temperatures $20,40,60$, 80 , and $100^{\circ} \mathrm{C}$ are applied on the beam anchor and different expansion positions of the free-end of microcantilever are identified. To limit the influence of temperature on the AFM tip fabricated from $\mathrm{Si}_{3} \mathrm{~N}_{4}$, after each measurement, the AFM piezo-table is moved to the zero position that is the initial starting location of the scanning process. The temperature increases to the next value when the AFM piezo-table is in this position. The AFM probe used in experiments is NSC36/Si3N4/AlBS/15(B) with a tip height of $25 \mu \mathrm{m}$. The lever of AFM probe, optically monitored during scanning, is suspended at $25 \mu \mathrm{m}$ above sample. Based on these aspects, the temperature influence on the lever of AFM probe is considered relatively small.

The sample illustrated in Fig. 3 (top view) was manufactured in the Laboratory for Analysis and Architecture of System in Toulouse (France). The material used to fabricate this microcantilever is gold and the structure was fabricated in 10 lithography and deposition steps with a gap between flexible part and substrate of $3 \mu \mathrm{m}$ (Pustan et al. 2007). Metals with high conductivity, such as aluminum or gold are used to fabricate the thermal components that operate at low temperature. The gold material has high

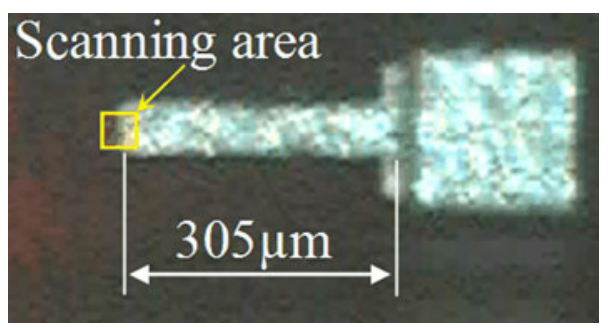

Fig. 3 Plan view of the microcantilever used in experimental investigations and the scanning area 
thermal efficiency and short thermal time constant of relatively low temperature $\left(<200^{\circ} \mathrm{C}\right)$.

The geometrical dimensions of the studied sample are the following:

- Of the flexible part of beam length of $305 \mu \mathrm{m}$, width of $57 \mu \mathrm{m}$, and thickness of $3 \mu \mathrm{m}$;

- Of the beam anchor-quadrate cross-section with side of $200 \mu \mathrm{m}$ and the thickness of $6 \mu \mathrm{m}$.

The experimental tests were performed at Technical University of Cluj-Napoca (Romania), in Machine Elements and Tribology Department using an AFM NT-206 device. The AFM investigations were developed in a clean room and the measurements were repeated 10 times. The relative difference between results of the axial expansions measurements is about $\pm 1.5 \%$ and the average results were considered. An initial calibration of the AFM scanning mode was performed using a typical surface structure (test grating) with known geometry (step size and height).
The plan view AFM images of sample, presented in Figs. $4 \mathrm{a}$ and $5 \mathrm{a}$, give information about the thermal axial expansion. Figure 4a shows a scanning map of the free-end of microcantilever at $20^{\circ} \mathrm{C}$. The initial position of the freeend of microcantilever in horizontal direction is at $10.8 \mu \mathrm{m}$ on the scanning map. The beam is on the right side of the cursor position that corresponds to $x=10.8 \mu \mathrm{m}$ as presented in Fig. 4b. To change the temperature of microcantilever a hotplate is positioned under the beam anchor. The position of free-end of sample is moving in the left direction during temperature increasing. At $100^{\circ} \mathrm{C}$ the position of free-end is moved to $10.1 \mu \mathrm{m}$ due to thermal expansion (Fig. 5b). The initial position of the free-end of microcantilever at $20^{\circ} \mathrm{C}$ being $10.8 \mu \mathrm{m}$ (Fig. 4b). The tendency of investigated microcantilever to change in volume as response to change in temperature is known as thermal expansion. The difference between both measurements at 20 and $100^{\circ} \mathrm{C}$ gives us the axial thermal expansion of microcantilever in $x$ direction which is equals to $0.7 \mu \mathrm{m}$.
Fig. 4 AFM image of the freeend of microcantilever at $20^{\circ} \mathrm{C}$ : a plan view AFM image, b sample topography

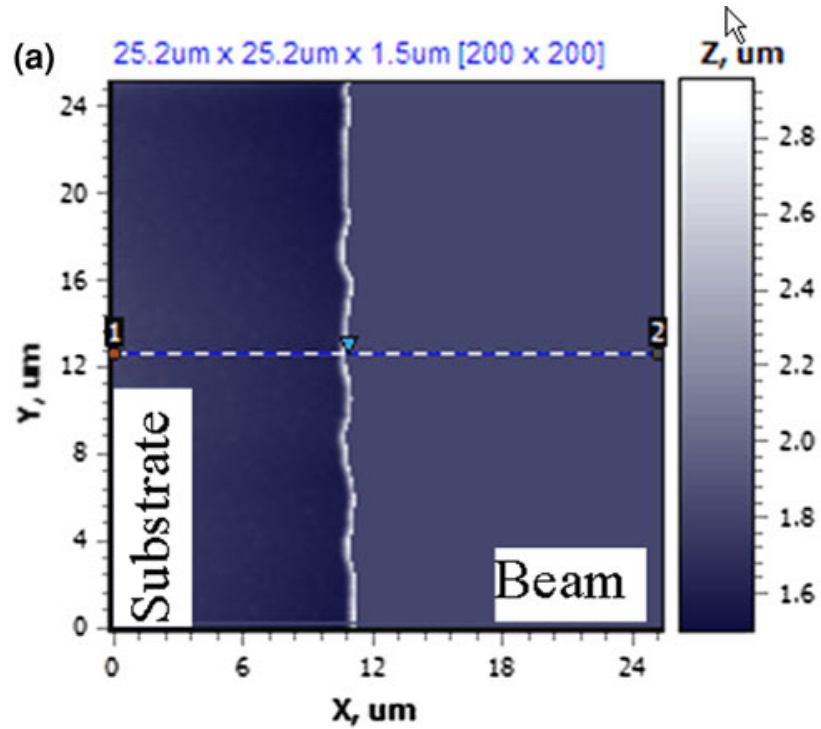

(b) Section $1-2$

[a]

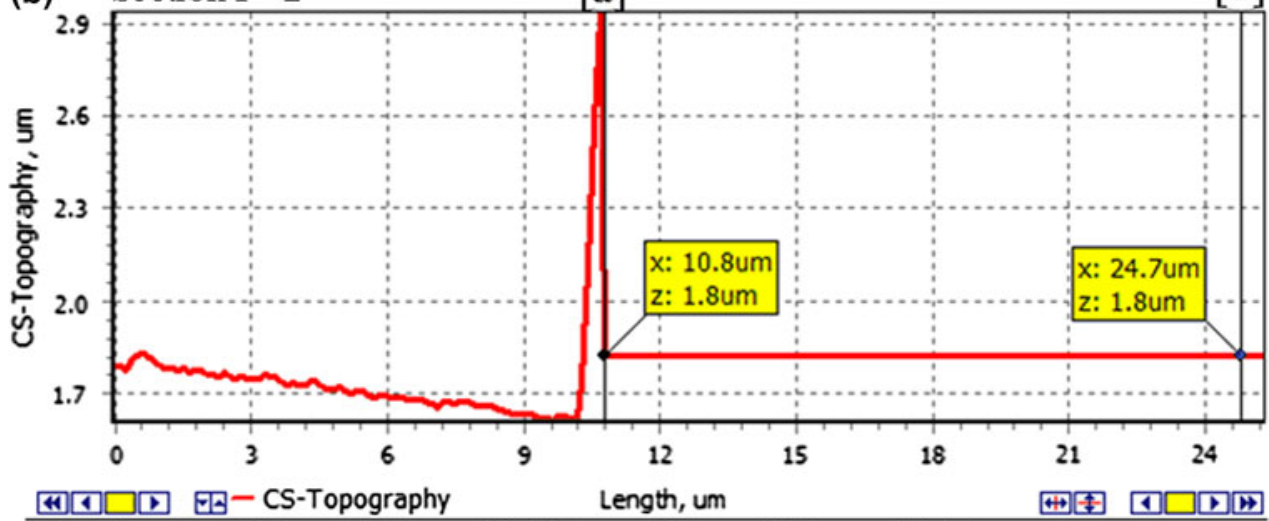

Absolute values: [a]-> $x=10.8$ um; $z(1)=1.8 u m ; \quad[b]->x=24.7 u m ; z(1)=1.8 u m$; Difference between markers: $d x=13.9 u m ; d z(1)=0.0 u m$;

Difference between first two lines: $x[a]=10.8 u m, d z[a]=0.0 u m ; x[b]=24.7 u m, d z[b]=0.0 u m$ 
Fig. 5 AFM image of the free-end of microcantilever at $100^{\circ} \mathrm{C}$ : a plan view $\mathrm{AFM}$ image, b sample topography
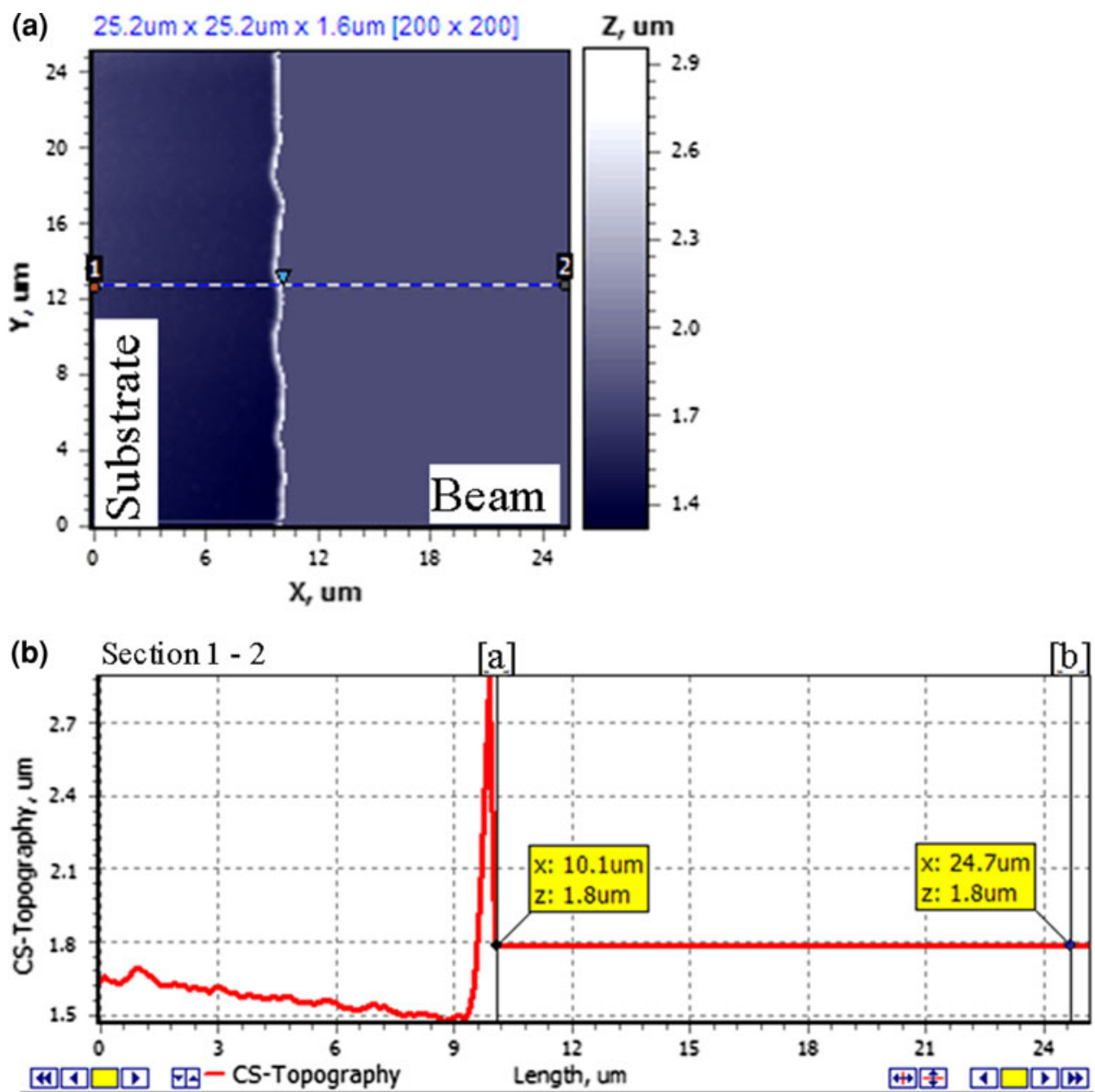

Absolute values: [a]-> $x=10.1$ um; $z(1)=1.8 u m ; \quad[b]->x=24.7 u m ; z(1)=1.8 u m$; Difference between markers: $d x=14.6 u m ; d z(1)=0.0 u m$;

Difference between first two lines: $x[a]=10.1 u m, d z[a]=0.0 u m ; x[b]=24.7 u m, d z[b]=0.0 u m$
Conforming to $z$ markers from Figs. $4 \mathrm{~b}$ and $5 \mathrm{~b}$, we can observe that there is no thermal bending deflection in vertical direction.

Figure 6 shows the experimental thermal effect on the axial displacement of the microcantilever free-end as a function of temperature, considering that the initial position of microcantilever corresponds to a temperature of $20^{\circ} \mathrm{C}$. During experimental tests, the length of microcantilever increases by $0.23 \%$ when the temperature of hotplate achieves $100^{\circ} \mathrm{C}$.

Axial expanding of the thermally actuated microcantilever (including expansion of the flexible part and of the beam anchor) produces the resulting thermal displacement of the free-end of sample. At low temperature $\left(20-40^{\circ} \mathrm{C}\right)$, the relation between the axial expansion of sample and temperature is no more linear and a lower slope is observed. This may be due to default and prestress appearing during fabrication process (McCarthy et al. 2006). A stress gradient occurs over the microcantilever during the microfabrication process. The residual stress

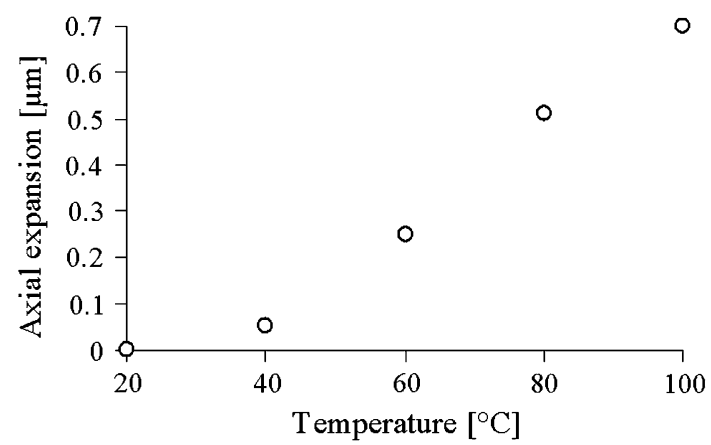

Fig. 6 Experimental variation of thermal axial displacement of the free-end of $305 \mu \mathrm{m}$ length gold microcantilever as a function of temperature

leads to an actuation temperature offset. Because, an initial increase in temperature is required to overcome the stress gradient imparted during microfabrication the axial expansion of the microcantilever as a function of temperature is characterized by a lower slope at low temperature $\left(20-40^{\circ} \mathrm{C}\right)$ as it is presented in Fig. 6. 
3.2 Bending stiffness measurement of a microcantilever using atomic force microscope

The stiffness is a fundamental property of elastically deformable mechanical microcomponents and micromechanisms for which static, modal or dynamic responses need to be evaluated (Lobontiu and Garcia 2004).

To measure the stiffness of the investigated sample, an AFM is used and the following main steps have to be performed (Pustan et al. 2007):

(a) The calibration of AFM parameters in normal direction for operation in contact mode;

(b) Determination of the real stiffness of AFM probe using a specific fabricated microspring with a wellknown stiffness. For AFM experimental tests of the investigated microcantilever, the type of the AFM probe chosen (manufactured by MicroMasch) is NSC36/Si3N4/AlBS/15(B) with the stiffness between 0.45 and $5 \mathrm{~N} / \mathrm{m}$, and the resonant frequency between 95 and $230 \mathrm{kHz}$. The real stiffness of the AFM probe obtained after calibration is $1.52 \mathrm{~N} / \mathrm{m}$.

(c) Verification of the temperature effect on the stiffness of AFM probe using the AFM dynamic mode. In this step, the AFM probe is oscillated close to the heating stage and the resonant frequency is monitored at different temperatures. The change in stiffness of AFM probe as a function of temperature can be estimated based on its detected resonant frequency. The measured resonant frequency of the AFM probe is $152.41 \mathrm{kHz}$ and it was not changed when the temperature increases from 20 to $100^{\circ} \mathrm{C}$. The material of AFM probe is $\mathrm{Si}_{3} \mathrm{~N}_{4}$ and the height of tip is $25 \mu \mathrm{m}$. During testing, the lever of AFM probe is suspended at $25 \mu \mathrm{m}$ above sample and the temperature effect on the AFM probe is diminished.

(d) Determination of the bending displacement of sample. The first step of the AFM measurements corresponds to the bending of AFM probe $Z_{\text {def }}$ and sample $Z_{\text {sample }}$ as illustrated in Fig. 7. The bending displacement of sample $Z_{\text {sample }}$ is determined based on the

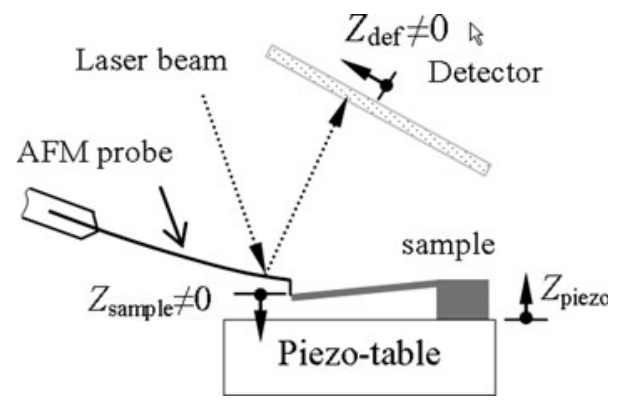

Fig. 7 Bending of AFM probe and sample controlled displacement of piezo-table $Z_{\text {piezo }}$ and the monitored deflection of AFM probe $Z_{\text {def }}$ as:

$Z_{\text {sample }}=Z_{\text {piezo }}-Z_{\text {def }}$

(e) Determination of the bending stiffness of sample. The stiffness of AFM probe $k_{A F M}$ is accurately known. Using the deflection of the AFM probe from the second step of the AFM measurements (Fig. 8) and the stiffness of the AFM probe, the acting force $F$ can be computed. This force and the displacement $Z_{\text {sample }}$ described by (9) give us the stiffness of sample as:

$k_{\text {sample }}=\frac{F}{Z_{\text {sample }}}$

Using the AFM technique to measure the bending, the stiffness of investigated microcomponent is estimated for different temperatures. While the experimental stiffness of microcantilever is $1.11 \mathrm{~N} / \mathrm{m}$ at $20^{\circ} \mathrm{C}$, the stiffness decreases by about $18 \%$ when the temperature increases to $100^{\circ} \mathrm{C}$ as shown in Fig. 9 due to the increase of the sample dimensions and decrease of the modulus of elasticity.

Indeed the thermal expansion changes the size of the microcomponent and the intrinsic mechanical behavior of materials (Tabor 1981; Lior and Izhak 2004; Stainier and Ortiz 2010). Using (8) and based on the force and displacement AFM measurements, the variation of Young's modulus for different temperatures is determined. The experimental value of Young's modulus at $20^{\circ} \mathrm{C}$ is 81.8 $\mathrm{GPa}$ and its value decreases to $66.2 \mathrm{GPa}$ if the temperature increases to $100^{\circ} \mathrm{C}$. The coupling of the strain field to a temperature field provides an energy dissipation mechanism that allows the system to relax and in a case of the investigated microcantilever under bending displacement, the relaxation strength to be considered is that of Young's modulus (Lifshitz and Roukes 2000; Lobontiu 2007) as:

$\Delta_{E}=\frac{E_{a d}-E}{E}=\frac{E \alpha^{2} \Delta T}{C_{p}}$ 


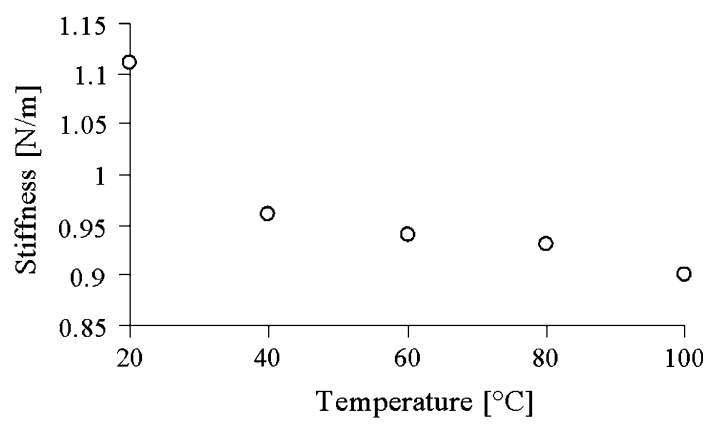

Fig. 9 Experimental variation of the microcantilever bending stiffness as a function of temperature

where, $E_{a d}$ is the unrelaxed value of Young's modulus, $E$ its relaxed value and $C_{p}$ is the heat capacity per unit volume at constant pressure. These effects have an influence in particular on stiffness (Fig. 9) but also on the friction coefficient as presented in the next section.

\subsection{Variation of adhesion and friction forces} as a function of temperature

Three basic characteristics are involved in the friction of dry solids (Tabor 1981; Lior and Izhak 2004; Adams et al. 2003):

- The true area of contact between rough surfaces

- The type and strength of bond formed at the interface where the contact occurs

- The shearing and rupturing characteristics of the material in and around the contact regions which can be influenced by temperature.

To estimate the friction coefficient, friction force should be divided by the sum of applied normal load $F_{\mathrm{AFM}}$ (given by the bending of AFM probe) and intrinsic adhesive force $F_{\text {adh }}$ (Bhushan 2003; Kapila et al. 2006; Riedo and Brune 2003; Schonherr et al. 2008).

The adhesive effect between AFM probe $\left(\mathrm{Si}_{3} \mathrm{~N}_{4}\right)$ and sample can be evaluated using the force spectroscopy mode of AFM. The force spectroscopy AFM curves provide the direct measurement of tip-sample interaction forces as a function of the gap between tip and sample. The adhesion between tip and sample is characterized by so-called pulloff or pull-out force. The pull-off force is related in current continuum contact mechanics model to the work of adhesion.

During experimental tests the sample is moved up and down (in and out of contact with the tip) and a dependence curve between displacements of piezo-table versus deflection of AFM probe is obtained. The AFM probe has a well-known stiffness and using the experimental AFM values, the dependence between force of AFM probe and displacement of piezo-table is computed. Figure 10 shows the adhesion effect between the AFM tip $\left(\mathrm{Si}_{3} \mathrm{~N}_{4}\right)$ and the investigated gold sample at $20^{\circ} \mathrm{C}$. Firstly, there is the bending deflection of AFM probe during loading (A-D). The unloading part of the force-displacement curve starts from position $\mathrm{D}$, the deflection of the AFM probe is decreased as the sample surface retracts from the tip. When the sample surface is further withdrawn from the tip, the AFM probe is deflected owing to adhesive forces. At position E, the elastic force in the AFM probe overcomes the adhesive forces and the tip snaps off from the surface (position F).

The adhesion force between the AFM tip $\left(\mathrm{Si}_{3} \mathrm{~N}_{4}\right)$ and the gold microcantilever is $21 \mathrm{nN}$ (Fig. 10). The measurements were performed under ambient conditions on the top surface of the cantilever anchor. The experimental variation of adhesive force between the AFM tip and the investigated gold microcantilever as a function of temperature is summarized in Fig. 11. A decrease by about $20 \%$ of the adhesive force when the temperature increases to $100^{\circ} \mathrm{C}$ is experimental determined. In the ambient environment, the condensed water will form a meniscus effect as the AFM tip approaches the sample surface, which leads to an increase of the friction force (Bhushan 2005). The meniscus force is a major contributor to the adhesive force. As the temperature increases, there is a decrease in adhesive force because of the lack of a meniscus contribution.

The friction coefficient is estimated by AFM measurements of frictional force. In that case, the two surfaces in contact are the tip of AFM probe and the sample (the top surface of the cantilever anchor). This measurement provides an index of friction behavior between two materials being in contact and in relative motion. The relative motion between tip and surface is realized by a scanner composed of piezoelectric elements, which move the material surface perpendicular to the tip of the AFM probe with a certain

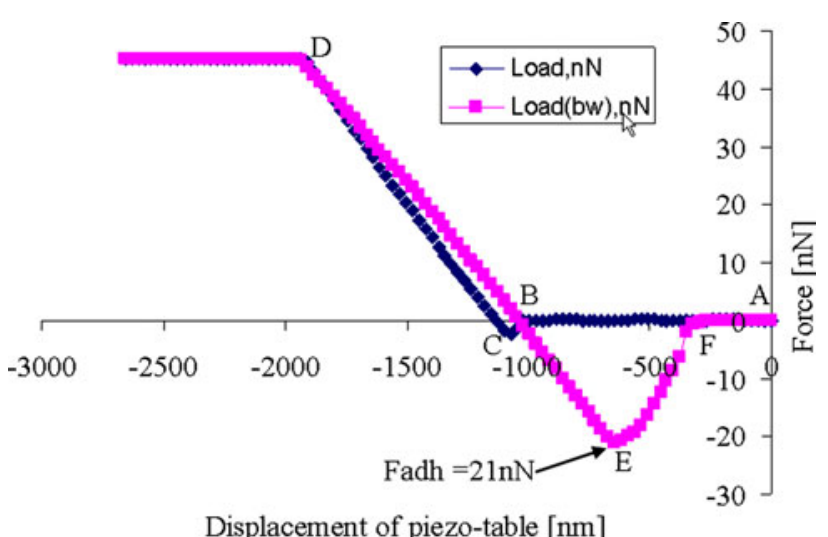

Fig. 10 Force versus AFM piezo-table displacement of a gold sample at $20^{\circ} \mathrm{C}$ 


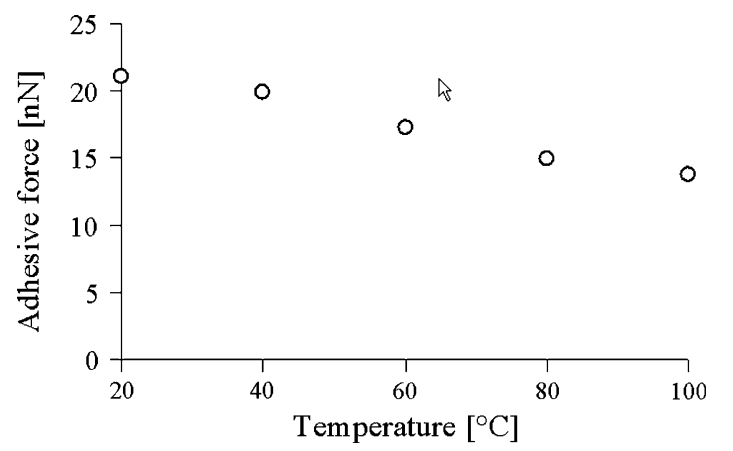

Fig. 11 Experimental variation of adhesive forces as a function of temperature between sample (gold) and the tip of AFM probe $\left(\mathrm{Si}_{3} \mathrm{~N}_{4}\right)$

periodicity as shown in Fig. 12. The scanner can also be extended or retracted in order to modify the normal force applied to the surface. This force gives information on the bending deflection of AFM probe. If the normal force increases while scanning because the surface is not flat, the scanner is retracted by a feedback loop. On the other hand, if the normal force decreases, the surface is brought closer to the tip by extending the scanner. The relative sliding of the AFM probe tip on the top surface of the beam anchor is influenced by friction. The lateral force, which acts in the opposite direction of the scan velocity, causes torsion of the AFM probe. Using a detector as illustrated in Figs. 7 and 8, we can determine the normal force (given by the bending deflection of AFM probe and its stiffness) and the lateral movements of the AFM probe during scanning.

The analysis of tribological characteristics of a thermal microcomponent fabricated from gold, using the AFM lateral force mode is now presented. The AFM probe, fabricated from $\mathrm{Si}_{3} \mathrm{~N}_{4}$, is moving toward and backward in the lateral direction on the top surface of the beam anchor (Fig. 12). Rotational (torsion) deflection $d z$ of AFM probe is measured, and after calibration (Pustan et al. 2007; Schonherr et al. 2008) the friction force is evaluated with the following well-known formula that is computed based on the torsion beam theory:

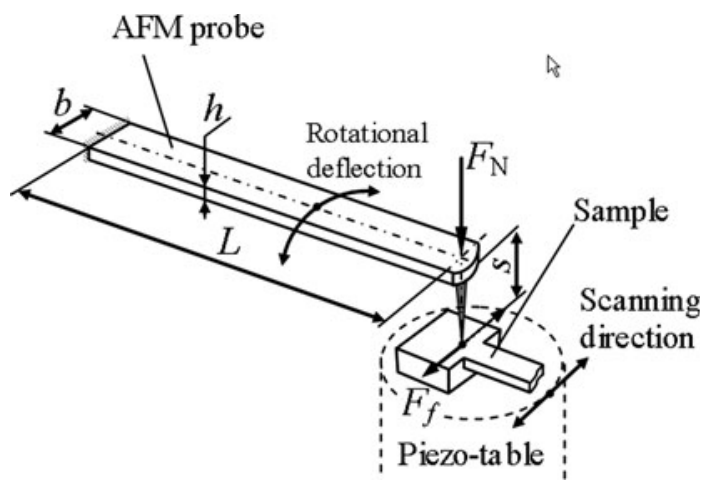

Fig. 12 Scanning principle and geometrical dimensions of an AFM probe
$F_{f}=\frac{d z \cdot r \cdot G \cdot h^{3} \cdot b}{L^{2} \cdot s}$

where, $d z$ is the calibrated deflection of AFM probe $(\mathrm{nm})$, $r=0.33, G$ shear modulus of the cantilever material, $L$ length of cantilever, $h$ thickness of AFM probe, $b$ width of AFM probe, and $s$ is the height of tip of AFM probe (Fig. 12).

Using the detected rotational deflection of AFM probe, the friction force between the gold sample and the tip of AFM probe $\left(\mathrm{Si}_{3} \mathrm{~N}_{4}\right)$ is evaluated for different temperatures based on (12). Knowing the total normal force $F_{N}$ (as a sum between normal force gives by bending of AFM probe $F_{\mathrm{AFM}}$ and the adhesive force $\left.F_{\mathrm{adh}}\right)$ and using friction forces, the friction coefficient is computed. Figure 13 shows the variation of the friction coefficient as a function of temperature. From this figure, it is seen that the friction coefficient decreases nonlinearly with increasing temperature. At high temperature, desorption of water from environmental humidity leads to decrease the meniscus force (which is a major contributor to the adhesive force), and the friction between the AFM tip and the investigated sample (Bhushan 2005; Kapila et al. 2006; Liu and Bhushan 2003; Riedo and Brune 2003). As the temperature is increased, the force needed to shear the contacting junctions where adhesion occurs decreases, respectively, and the tip jump the surface potential barriers more easily, resulting in lower shear strength and hence lower friction force and friction coefficient (Kapila et al. 2006).

\section{Finite element analysis of thermal expansion of a cantilever}

Finite element simulations are widely used to model MEMS thermal actuator in order to provide temperature, expansion and stress distributions. To evaluate the distribution of temperature in a gold microcantilever and to determinate the maximum value for the axial expansion of

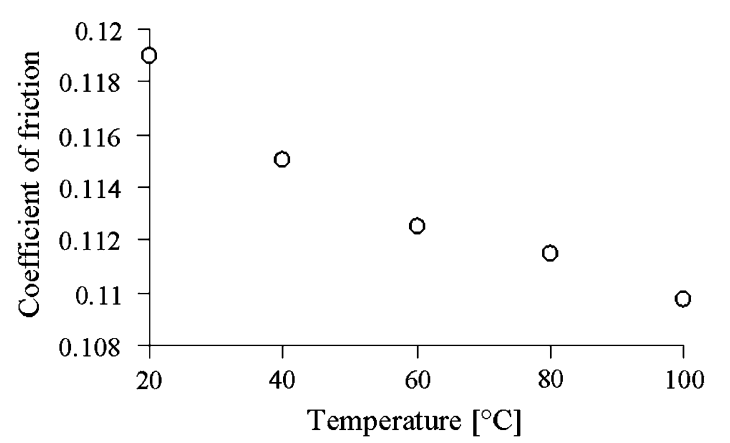

Fig. 13 Experimental variation of friction coefficient as a function of temperature between sample (gold) and the tip of AFM probe $\left(\mathrm{Si}_{3} \mathrm{~N}_{4}\right)$ 


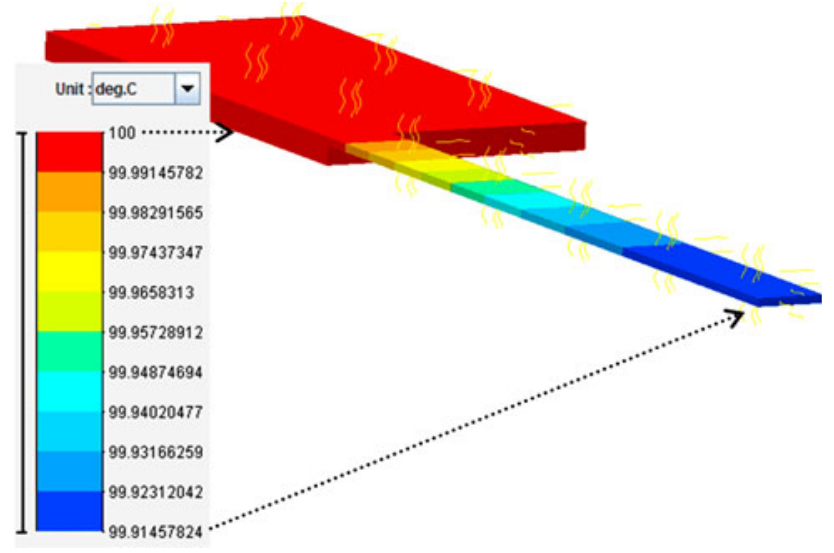

Fig. 14 Thermal field distribution of a gold microcantilever

the free-end of beam, finite element analysis (FEA) is carried out using a commercial version of Oofelie::Multiphysics Simulation Software.

Considering only the half of the beam due to the problem symmetry the distribution of the thermal field inside of structure was computed. Figure 14 shows the distribution of the thermal field in the investigated microcantilever when the temperature applied on anchor is $100^{\circ} \mathrm{C}$. The temperature at the free-end of the flexible part of beam is about $99.91{ }^{\circ} \mathrm{C}$.

Figure 15 shows the result of finite element analysis of the axial expansion of the investigated microcantilever when the temperature increases to $100^{\circ} \mathrm{C}$. The axial expansion of the free-end of the flexible part of microcantilever is about $0.68 \mu \mathrm{m}$ and it is close to the experimental results, which are plotted in the Fig. 6. The difference between FEA results and the experimental results are affected by the following: the accuracy of experimental tests which depends on the testing conditions, on the initial calibration of the AFM device and the sensitivity of the AFM probe; the Young's modulus of

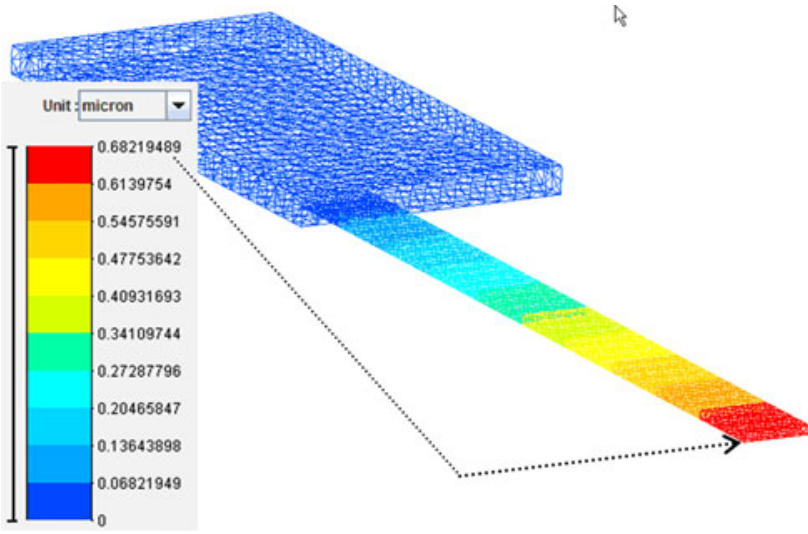

Fig. 15 Finite element analysis of the axial expansion of a gold microcantilever at $100^{\circ} \mathrm{C}$
$82 \mathrm{GPa}$ used in FEA was taken from literature (http:// www.memsnet.org/material) and it can differ from the experimental Young's modulus; the differences between theoretical dimensions of sample and the real dimensions (in FEA the theoretical dimensions are used).

\section{Conclusions}

This paper shows that the use of AFM is effective to study the mechanical behavior of microcomponents as a function of temperature. The axial thermal expansions, bending deflections as well as bending stiffness versus temperature of a thermal actuated microcantilever were successfully identified. The AFM operating in lateral force mode is also useful to measure the friction force at sliding (scanning) on gold microcomponent. The effect of increasing temperature on friction coefficient between the p-type silicon tip of the AFM probe and a gold sample was identified. The meniscus effect has a big influence on the adhesive and friction forces. A decrease in adhesive force between the tip of AFM probe $\left(\mathrm{Si}_{3} \mathrm{~N}_{4}\right)$ and the gold cantilever as the temperature increases, based on the lack of the meniscus effect, was experimental determined. As the temperature increases, resulting in lower shear strength and decreasing of the coefficient of friction. Finite element analysis has been used to analyze the distribution of the temperature and to simulate the axial expansion of a gold microcantilever. The finite element analysis results are in good agreement with the experimental results.

Acknowledgments This work was supported by a grant of the Romanian National Authority for Scientific Research, CNCS-UEFISCDI, project number PN-II-RU-TE-2011-3-0106. The first author wishes to explain his gratitude to Open-Engineering Co for their support in modeling and finite element analysis.

\section{References}

Adams G, Müftü S, Azhar MN (2003) A nano-scale multi-asperity contact and friction model. ASME J Tribol 125:700-708

Bhushan B (2003) Adhesion and stiction: mechanisms, measurement techniques, and methods for reduction. J Vac Sci Technol B21(6):2262-2296

Bhushan B (2005) Handbook of nanotribology and nanomechanicsan introduction. Springer, Berlin

Chu LL, Gianchandani YB (2003) A micromachined 2D positioner with electrothermal actuation and sub-nanometer capacitive sensing. J Micromech Microeng 13:279-285

Geisberger AA, Sarkas N, Ellis M, Skidmore G (2003) Electrothermal properties and modeling of polysilicon microthermal actuators. J Microelectromech Syst 12:516-523

Kapila V, Deymier PA, Raghavan S (2006) Molecular dynamic simulations of friction between alkylsilane monolayers. Modelling Simul Mater Sci Eng 14:283-297

Lifshitz R, Roukes ML (2000) Thermoelastic damping in micro and nanomechanical systems. Phys Rev B 61:5600-5609 
Lior K, Izhak E (2004) A static friction model for elastic-plastic contacting rough surfaces. ASME J Tribol 126:34-40

Liu H, Bhushan B (2003) Nanotribological characterization of molecularly thick lubricant films for applications to MEME/ NEMS by AFM. Ultramicroscopy 97:321-340

Lobontiu N (2007) Dynamics of Microelectromechanical System. Springer, NY

Lobontiu N, Garcia E (2004) Mechanics of microelectromechanical systems. Cornell University, Ithaca

McCarthy M, Tiliakos N, Modi V, Frechette LG (2006) Thermal buckling of eccentric microfabricated nickel beam as temperature regulated nonlinear actuators for flow control. J Sens Actuators A 134:37-46

Park JS, Chu LL, Oliver AD, Gianchandani YB (2001) Bent-beam electrothermal actuators. Part II linear and rotary microengines. J Microelectromech Syst 10:255-262

Paryab N, Jahed H, Khajepour A (2009) Creep and fatigue in singleand double-hot arm MEMS thermal actuators. J Fail Anal and Preven 9:159-170

Pustan M, Ekwinski G, Rymuza Z (2007) Nanomechanical studies of MEMS structures. Int J Mater Res 5:384-388
Rebeiz GM (2003) RF MEMS-Theory design and technology. Wiley, Hoboken

Riedo E, Brune H (2003) Young modulus dependence of nanoscopic friction coefficient in hard coating. Appl Phys Lett 83:1986-1988

Schonherr H, Tocha E, Vasco J (2008) Friction and surface dynamics of polymers on the nanoscale by AFM. Top Curr Chem 285: $103-156$

Stainier L, Ortiz M (2010) Study and validation of a variational theory of thermo-mechanical coupling in finite visco-plasticity. Int J Sol Str 47:705-715

Tabor D (1981) Friction-the present state of our understanding. ASME J Lubrication Tech 103:169-179

Tadayon MA, Sayyaadi H, Jazar Nakhaie G (2006) Nonlinear modeling and simulation of thermal effects in microcantilever resonators dynamic. J Phys Confer Series 34:89-94

Yang P, Stevenson M, Lai Y, Mechefske C, Kujath M, Hubbard T (2008) Design, modeling and testing of a unidirectional MEMS ring thermal actuator. Sensor Actuators A143:352-359

Zhu Y, Corigliano A, Espinosa D (2006) A thermal actuator for nanoscale in situ microscopy testing: design and characterization. J Micromech Microeng 16:242-253 